\title{
An approach of spectrum analysis to the stock prediction
}

\author{
JAMES L. LUO \\ jameslgluo@hotmail.com
}

\section{Introduction}

\section{A. Efficient Market Hypothesis (EMH)}

Is the stock prediction possible? The answers differ from theory to theory. In general, there are two points of view: micro one and macro one. Studies of the former are based on game theory, but usually do not concern the question. The latter has two opposite methodologies: modern finance (negative answer), including MM theory, MPT, CAPM, APT, EMH, BS formula etc., and behavioral finance (positive answer). There are other theories between them, such as $\mathrm{ARCH}, \mathrm{GARCH}$, Cointegration, R/S etc., that belong to stochastic time series analysis. All of these theories are related to asset pricing. We will briefly introduce some of them, but mainly focus on EMH to show its intrinsic contradiction, that is, the major flaw of modern finance.

Fama's EMH assumes that "security price at any time 'full reflect' all available information.", 1 thus "the series of price changes has no memory, that is, the past cannot be used to predict the future in any meaningful way.", , which refers to chart reading of weak-efficient form, or technical analysis. Fama also argues that semi-strong and strong form tests almost support EMH, i.e. the fundamental analysis and insider trading can not make excess profits as well - like the bible says, you do not even know what will happen tomorrow. Hence, as Fama argues, "in a random-walk market the security analysis problem of the average investor is greatly simplified." ${ }^{3}$ : the only rational strategy of investment is some buy-and-hold policy, i.e. passive investment, e.g. ETFs.

Is the world so simple and perfect? Luckily, Fama put the word "average" in front of "investor", otherwise if all investors take this strategy, who will sell? Fama does admit there exist superior analysts (e.g. Warren Baffet) who gain more than those investors who follow the buy-and-hold policy, but he claims that if there are enough superior analysts to capitalize on chances of "wrong" pricing, they will make fundamental analysis useless for average ones. But how many such analysts are necessary and how to indentify thses analysts? For the first question, Fama thinks it is impossible to give a firm answer; and for the second, he proposes a simple criterion: a superior analyst is one whose gains over many periods of time are consistently greater than those of the market. ${ }^{4}$ Is such test simple? Of course not - it takes

\footnotetext{
${ }^{1}$ Eugene F. Fama, Efficient Capital Market: A Review of Theory and Empirical Work, Journal of Finance, Volume 25, Issue 2, 1969, P.383.

${ }^{2}$ Fama, The Behavior of Stock-Market Prices, Journal of Business, Volume 38, Issue 1, 1965, P.34.

3 Id., P.40.

${ }^{4}$ Id.
} 
time and cost much, in particular considering verifications of data and rights of privacy. Of course Fama take it for granted that the number of superior analysts is not above some "minimum acceptable" level so that the EMH holds in reality. ${ }^{5}$ However, how to define such level? For some observable dependencies of empirical data, Fama also begs the questions, ${ }^{6}$ which seems to be more a belief than a research.

Morever, empirical tests of semi-strong and strong form are almost invalid in terms of mathematics since they are hard to quantitate, thus the only possible verification lies in the weak form. Fama's paper in 1965 investigates this test. In this paper he reiterates that the random walk model "involves two separate hypotheses: 1) successive price changes are independent, and 2) the price changes conform to some probability distribution." ", and argues "In the general theory of random walks the form or shape of the distribution need not be specified." 8 , which refers to the generalized central limit theorem (GCTL). Since the leptokurtosis with fat tails are usually observed in empirical data, ${ }^{9}$ Fama concludes that stable Paretian distributions with infinite variances are a better description of distributions of daily returns than the normal ones with finite variances. In such way, the assumption of independent and identical distribution of random variables still holds, since the normal one is just a specific case of stable distributions when the characteristic exponent $\alpha=2{ }^{10}$

However, italic "separate" actually implies that EMH is unverifiable. On the one hand, stable Paretian distribution is not the only possibility accounting for the leptokurtosis with fat tails, that may result from mixtures of normal distributions with different variances, ${ }^{11}$ i.e. non-identical distributions of random variables; or dependence, i.e. price changes have memory, which is deliberately ignored by Fama; or many other kinds of distributions. For

\footnotetext{
${ }^{5}$ Id., P.35.

${ }^{6}$ a) Fama, Efficient Capital Market: A Review of Theory and Empirical Work, P.396: "For example, one departure from the pure independence assumption of the random walk model has been note... large daily price changes tend to be followed by large daily changes. The signs of the successor changes are apparently random, however, which indicates that the phenomenon represents a denial of the random walk model but not of the market efficiency hypothesis... sometimes the initial price will overadjust to the information, and other times it will underadjust." P.414: "the dependence shows up as serial correlations that are consistently positive but also consistently close to zero, and as a slight tendency for observed numbers of runs of positive and negative price changes to be less than the numbers that would be expected from a purely random process... But any system (like the filters) that attempt to turn short-term dependence into trading profits of necessity generate so many transactions that their expected profits would be absorbed by even the minimum commissions (security handling fees) that floor traders on major exchanges must pay."

b) P.415: “... Niederhoffer-Osborne [32] finding of a tendency toward excessive reversals... however, there seems to be no way it can be used as the basis of a prifitable trading rule. As they rightly claim, their results are a strong refutation of the theory of random walks... but they do not constitute refutation of the economically more relevant 'fair game' efficient markets model."

c) Fama, The Behavior of Stock-Market Prices, P.37: "Even in a situation where there are dependencies in either the information or the noise fenerating process, however, it is still possible that there are offsetting mechanisms in the market which tend to produce independence in price changes for individual common stocks... If there are enough of these sophisticated traders, they may tend to prevent these 'bubbles' from ever occurring. Thus their actions will neutralize the dependence in the noise-generating process, and successive price changes will be independent."

7 Id., P.35, P.40-41, P.89.

${ }^{8}$ Id., P.41.

9 Id., P.49-50: "In simpler terms, if the population of price changes is strictly normal, on the average for any given stock we would expect an observation greater than 4 standard deviations from the mean about once every fifty years. In fact observations this extreme are observed about four times in every five-year period. Similarly, under the Gaussian hypothesis for any given stock an observation more than 5 standard deviations from the mean should be observed about once 7,000 years. In fact such observations seem to occur about once every three to four years." ${ }^{10}$ Id., P.43.

11 Fama, Efficient Capital Market: A Review of Theory and Empirical Work, P.399-400, footnote 23.
} 
independent and identically distributed random variables (IID), stable Paretian distribution is an attractor of those with infinite variances by GCTL, and vice versa. But long or fat tails do not necessarily lead to stable Paretian distributions as Fama himself states: "It is possible that there are long-tailed distributions with finite variances [as mixtures of normal distributions] that could also be used to describe the data." ${ }^{\prime 2}$ The empirical test will be invalid if the data has an observable departure from the normal distribution, let alone for large differencing intervals, the low frequency of tails means much less data and much larger errors. On the other, as long as the condition of finite variance is satisfied, normal distribution will be a stable domain of attraction by the central limit theorem, or a limit distribution under addition, and vice versa, i.e. an one-to-one correspondence between normality and IID with finite variance, or random walk, and it must be a martingale (proof omitted) as Fama states. ${ }^{13}$ Thus, there is only one normal distribution, while long-tailed distributions differ from one to one. Thus, only the results quite approximating normal distribution in most tests can provide strong empirical evidence in support of the assumption of independence or martingale, otherwise the weak form of EMH could not be verified in practice, just as Continuum Hypothesis in theory.

Fama's vagueness are partly due to his deep concern about the specification of the distribution shape from various points of view, for example, variance is defined as the risk of investment in modern finance. But he knows the fact that explicit expressions for the density functions of stable Paretian distributions are usually unknown ${ }^{14}$ since in general the Fourier inverse transform of its (logarithm) characteristic function $(\log ) f(\mathrm{t})$ has no explicit solution: ${ }^{15}$

$$
\log f(t)=\log \int_{-\infty}^{+\infty} \exp (i u t) \mathrm{d} P=i \delta t-\gamma|t|^{\alpha}\left[1+\frac{i \beta t}{|t|} \tan \left(\frac{\alpha \pi}{2}\right)\right] .
$$

Moreover, infinite variance means unlimited risk of investment in terms of modern finance, which is unreasonable in reality. Thus, Fama later gives up the approach of stable Paretian distribution though he complains that "Economists have, however, been reluctant to accept these results, primarily because of the wealth of statistical techniques available for dealing with normal variables and relative paucity of such techniques for non-normal stable variables." 16 This viewpoint does make sense. For instance, as a student of Fama, Myron Scholes yet believed in the normal distribution of price changes since it gives a finite and constant variance of historical data, i.e. volatility, a fundamental of Black-Scholes-Merton option pricing formula (obviously, BS formula can not stand on stable Paretian distribution). Such credo was also the philosophy of Long-Term Capital Management (LTCM), though neither Merton or Scholes was involved in arbitrage trading or creating models at the firm. ${ }^{17}$ They certainly knew the problem of long tails, but without appropriate statistical tools, non-normal distribution meant nothing for them. Thus, in contrast to Fama's pure empirical works, Merton and Scholes built their financial theory upon the exact normality, that was

\footnotetext{
${ }_{12}$ Fama, The Behavior of Stock-Market Prices, Journal of Business, Volume 38, Issue 1, 1965, P.97.

13 Fama, Efficient Capital Market: A Review of Theory and Empirical Work, P.385-386.

${ }_{15}$ Fama, The Behavior of Stock-Market Prices, P.99.

15 Fama, Mandelbrot and the Stable Paretian Hypothesis, Journal of Business, Vol.36, No.4 (Oct., 1963), P.421.

${ }^{16}$ Fama, Efficient Capital Market: A Review of Theory and Empirical Work, P.399-400.

${ }^{17}$ Loger Lowenstein, When Genius Failed: the Rise and Fall of Long-Term Capital Management, Random House Trade Paperbacks, 2001, P.65.
} 
worth defending ${ }^{18}$ as a perfect belief, ${ }^{19}$ but in the name of efficient market. However, the imperfect tail finally killed LTCM in 1998, along with high leverages; and destroyed their second efforts in 2008 though the level of leverages were lowered - the assumption of normality was the key issue while the leverage just blew it up.

This does not deny, however, the fact that it is hard to make predictions through technical or fundamental analysis, which may be due to asymmetric information and limited rationality rather than perfect information and rationality as Fama's assumptions of (ii) and (iii). ${ }^{20}$ In fact, normal distribution may be the best approximation to real capital markets in most cases of some differencing intervals, e.g. day or week returns, but at least high-frequency or day trading is an exception, so do those financial crises. By analogy, EMH or normality works as the Geocentric model, but it will fail in extreme cases and have disastrous consequences. The dilemma of modern finance is that if we accept the existence of long tails, there will be a lack of useful analysis tools; or if we take the assumption of normality, the application will be imbedded with fatal bugs. For example, J. P. Morgan admitted the flaws of its Value-at-Risk (VAR) methodology, nevertheless, the firm and Wall Street continued to use such programs because they could not find any "persuasive alternatives", ${ }^{21}$ and so they were. Then, does there exist any alternative as the Heliocentric model? Let us show its possibility.

\section{B. Missed Information}

First, we take a look at the famous Monty Hall problem: there are three doors that behind one of them is a car and behind the other two there are goats, and the contestant wants to win a car. After the contestant chosses one door (but unopened), say door 1, the host opens another door showing a goat (the host knows everything), say door 2, then is it the contestant's advantage to switch to the remaining unopened door 3 ?

The question was presented at the TV show "Let's make a deal" by the host Monty Hall in 1975, on which Miss California Carol Merrill answered “yes", but a large number of audience including many mathematicians called or wrote to the TV station, said she was wrong. Indeed, Paul Erdos, as one of the most prolific and foremost mathematicians involved in probability, when was initially told the problem, yet at first was not convinced by the mathematical explanation and insisted his intuition: the two remaining unopened doors have the equal probabilities so that switching does not matter. ${ }^{22}$ The right answer, of course, is "yes",

\footnotetext{
${ }^{18}$ Id., P.75: "When Robert J. Shiller, an American economist, dared to suggest that markets were too volatile to fit the model of perfect markets, you could sense that for Merton, to entertain any doubt at all was to risk seeing the entire edific crumble: 'We need hardly mention the significance of such a conclusion. If Shiller's rejection of market efficiency is sustained, then serious doubt is cast on the validity of this cornerstone of modern financial econmic theory.",

${ }_{19}$ Robert C. Merton, Continuous-Time Finance, Basil Blackwell Inc., 1990, Preface: “As the Black-Scholes work was closing gates on fundamental research in these areas, it was simultaneously opening new gates: in applied and empirical study... Indeed, the mathematics of the continuous-time finance model contains some of the most beautiful applications of probability and optimization theory. But, of course, not all that is beautiful in science need also be practical. And surely, not all that is practical in science is beautiful. Here we have both."

${ }^{20}$ Fama, Efficient Capital Market: A Review of Theory and Empirical Work, P.387: "sufficient conditions... (i) there are no transaction costs in trading securities, (ii) all available information is costlessly available to all market participants, and (iii) all agree on the implications of current information for the current price and distributions of future price of each security."

${ }^{21}$ Lowenstein, When Genius Failed: the Rise and Fall of Long-Term Capital Management, P.76.

${ }^{22}$ Samuel Arbesman, Monty hall, Erdos, and our limited minds, https://www.wired.com
} 
because it will increase the chance of winning a car from $1 / 3$ of door 1 to $2 / 3$ of door $3-$ Erdos made a mistake of conditional probability. ${ }^{23}$ On the other hand, the explanation in terms of information asymmetry is more powerful: the host possess more information, then if he reveals something to reduce the uncertainty, we shall make use of it.

Do we miss any information in trading securities? Yes, it is volume. Any transactions have two and only two variables: price and quantity. However, almost all financial researches focus only on price changes in the time domain. Some techniques of chart reading takes volume into account at times, but it only concerns the total volume rather than its inner structure - the distribution of lot sizes in the frequency domain, a new concept inspired by my research on timbre evaluation of musical instruments, i.e. Luo's theory. ${ }^{24}$ The new approach is called Volume Spectrum Analysis (VSA), which constructs a specific "volume spectrum". It reveals asymmetric information of volume that does not contain in the distribution of day returns or longer, i.e. low-frequency trading (LFT), and can be combined with price changes to make predictions and enhance the overall efficiency of market.

A similar example in hand is high-frequency trading (HFT). The distribution of tick data (transaction by transaction data) shows its leptokurtosis and fat tails are much more remarkable than those of LFT, which strongly suggests (but as we have shown before, it does not prove) the dependence of price changes in the micro-structure of trading. HFT firms do capitalize on such asymmetric information by taking advantage of low latency access, liquidity rebates ${ }^{25}$ and specific algorithms etc., which is based on huge investment in human and technical resources. Many see the arbitrage of HFT as unethical and unfair to other investors. Some argue that HFT algorithms "spoof" through sending a large number of "fake" orders, but information distortion is always an inseparable part of the market - think about numerous "research reports". In fact, HFT helps the overall market by reducing bid-ask spread, creating high liquidity, and assisting on pricing etc. In addition, we will show in chapter III how to eliminate the effect of HFT in VSA since VSA only works on LFT.

Behavioral finance does recognize irrational actions in trading, e.g. cognitive biases such as herd instinct, self-rating, and loss aversion etc. However, it does not mean we are able to capitalize on such anomalies since such economic psychology as prospect theory or nudge theory is hard to be constructed as quantitative models. Moreover, some technical analysts consider behavioral finance as their theoretical basis, ${ }^{26}$ which puts behavioral finance in the trap of chart reading. On the other hand, stochastic time series analysis asserts that time series of returns follows a biased random process to modify the assumption of normality - it has time-varying volatility and nonstationarity. But it faces the same problem as traditional technical analysis since both of them rely only on price changes.

From a micro perspective, trading of capital market is a continuous double auction, which leads studies to noncooperative games. For one-side auction, the problem of optimal auction

\footnotetext{
${ }^{23}$ Using the sample space method to list all equal possibilities gives a more intuitive reasoning, proof omitted.

${ }^{24}$ Jame L. Luo, Introduction of Luo's Theory, 17 Mar. 2020, https://osf.io/yx29f/

${ }^{25}$ That means they do not need to pay transaction fees and even get rebates, which rejects Fama's argument in footnote 6, a).

${ }^{26}$ Charles D. Kirkpatrick \& Julie R. Dahlquist, Technical Analysis: The complete Resource for Financial Market Technicians, 2007, Upper Saddle River, NJ: Financial Times Press.
} 
design has been studied by Myerson ${ }^{27}$ and other researchers. In these papers, uniform, explicit utility function and Nash equilibrium of game theory are the basic methodologies. As Luo has shown, however, the former is arbitrary and the latter does not work in most economic actions because of the implied assumptions of unlimited ability of information collection and calculation. ${ }^{28}$ Such flaws remains and accounts for the difficulty of developments of double auction in succedent researches, e.g. Chatterjee and Samuelson ${ }^{29}$ Myerson and Satterthwaite, ${ }^{30}$ Friedman ${ }^{31}$ etc. And later researchers, e.g. Gode and Sunder, ${ }^{32}$ have to employ market experiments (computer simulation) to design strategy models for continuous double auction, which implies that the concept of static equilibrium does not apply to the dynamic asset pricing.

Our plan of the new approach based on information asymmetry is as follows. Chapter II presents the basic assumptions related to capital sizes of investors, and draws some corollaries that we will use in later proofs. In chapter III, we introduce the construction of volume spectrum, and prove the validity of its analysis in call auction and continuous trading. The possible applications, such as the explanation for bubbles, new approach to option pricing, prediction of financial crisis etc., are presented in chapter IV. In the last chapter, we discuss the relationship between information asymmetry and market efficiency.

\section{Basic assumptions}

We suppose that investment strategy is statistically subject to the capital size rather than modern finance's rationality or behavioral finance's irrationality. In general, a large order is more likely submitted by a large institute, while a small one may implies a retail investor. Whether for long or short positions, it is quite possible that at the time of entry large investors will set up higher stop-profit prices and lower stop-loss ones than those of small investors. For example of long position, say the former has $30 \%$ and $-15 \%$, the latter has $10 \%$ and $-5 \%$. The pattern is called stop setting, and its absolute value in percentage is called stop size. It does not mean investors close their positions exactly at stop prices every time - orders may be executed at any prices, but statistically they have such (volume weighted) average values. Moreover, we assume the closing prices are log-normally distributed. Some investors may not initialize explicit stop settings, but it is quite possible that they practice subconsciously in such way, since economic actions are subject to the capital sizes of investors - the large capital gives an advantage of possession and interpretation of information. And larger capital or stop size often means longer position time - it could be observed that small investors are

\footnotetext{
${ }^{27}$ Roger B. Myerson, Optimal Auction Design, Mathematics of Operations Research, 1981, Vol.6, pp.59-73.

28 James L. Luo, Is the Antitrust Policy Trustful? (I) - Strategy Mechanism of Lockefeller's Monopoly, 18 Jan. 2020, pp.2-3, https://osf.io/utrx6/

${ }_{29}$ Kalyan Chatterjee \& William Samuelson, Bargaining under In complete Information, Operation Research, 1983, Vol.31, pp.835-851.

${ }^{30}$ Roger B. Myerson \& Mark A. Satterthwaite, Efficient Mechanism of Bilateral Trading, Journal of Economic Theory, 1983, Vol.29, pp.265-281.

${ }^{31}$ Daniel Friedman, A Simple Testable Model of Double Auction Markets, Journal of Economic Behavior and Organization, 1991, Vol.15, pp.47-70.

${ }^{32}$ Dhananjay K. Gode \& Shyam Sunder, Allocative Efficiency of Markets with Zero Intelligence (ZI) Traders: Market as a partial Substitute for Individual Rationality, Journal of Political Economy, 1993, Vol.101, pp.119-137.
} 
apt to close their positions more avidly than larger ones when the market is in volatility - we will proof it soon after.

The stop sizes $Q$ depends on the order lots $x$, say $\left|E_{p}(x)\right|$ and $\left|E_{l}(x)\right|$, where $E_{p}$ is the (volume weighted) mean stop-profit price in percentage and $E_{l}$ is that of stop-loss; and their absolute prices are repectively $p_{0}\left(1+E_{p}(x)\right)$ and $p_{0}\left(1+E_{l}(x)\right)$, where $p_{0}$ is the opening price.

In a certain period of trading, suppose that a filled order is buy one with probability $q$ and sell one with probability $(1-q)$. For any enough long period, the overall behavior (net buying volume) of large investors is meant to be remarkably different from that of small ones. Thus assume $q$ also depends on the lot size of orders, i.e. $q=q(x)$.

Then for any enough long periods, information asymmetry of trading is stated as:

Assumption One: For $x \in N^{*}, a<x<b$, where $x$ is the lot size of a filled order, $a$ and $b$ are respectively the minimum and maximum lot size of trading; stop size $Q(x)$ is statistically monotone increasing and log-normally distributed.

Assumption Two: $x, a$ and $b$ are defined as before; for any filled order, its probability $q(x)$ of being a buy order is statistically monotone, which can be increasing or decreasing.

Three comments or details should be made about these assumptions: (1) detailed interpretations of Assumption Two are presented in the next chapter. (2) The structure of stop setting is symmetric. For example, for a filled buy order of lot size $x_{0}$, let its $E_{p}\left(x_{0}\right)=10 \%$ and $E_{l}\left(x_{0}\right)=-5 \%$; and then for a sell order of the same $x_{0}, E_{l}^{\prime}\left(x_{0}\right)=5 \%$ and $E_{p}^{\prime}\left(x_{0}\right)=-10 \%$, i.e. the short may buy shares back when the stock price rises to around 5\% or drops to around $-10 \%$. (3) For any one of orders, let $Y$ denote its continuous random variable of closing prices, $Y_{p}$ as that of stop-profit prices and $Y_{l}$ as that of stop-loss ones. Assume $\ln Y_{p} \sim N\left(\mu_{p}, \sigma_{p}^{2}\right)$ and $\ln \left|Y_{l}\right| \sim$ $N\left(\mu_{l}, \sigma_{l}^{2}\right)$. Thus, the probability of closing positions at a certain range of price could be represented by a probability density function (PDF):

$$
\begin{gathered}
Q(y, \mu, \sigma, c)=\left\{\begin{array}{l}
\frac{c_{p}}{y \sqrt{2 \pi \sigma_{p}^{2}}} \exp \left[-\frac{\left(\ln y-\mu_{p}\right)^{2}}{2 \sigma_{p}^{2}}\right], y>0 \\
\frac{c_{l}}{y \sqrt{2 \pi \sigma_{l}^{2}}} \exp \left[-\frac{\left(\ln |y|-\mu_{l}\right)^{2}}{2 \sigma_{l}^{2}}\right], y<0
\end{array}\right. \\
\sigma_{p}^{2}=\ln \left(1+R S D^{2}\left(Y_{p}\right)\right), \sigma_{l}^{2}=\ln \left(1+R S D^{2}\left(Y_{l}\right)\right) \\
\mu_{p}=\ln \left(\frac{E\left(Y_{p}\right)}{\sqrt{1+R S D^{2}\left(Y_{p}\right)}}\right), \mu_{l}=\ln \left(\frac{\left|E\left(Y_{l}\right)\right|}{\sqrt{1+R S D^{2}\left(Y_{l}\right)}}\right)
\end{gathered}
$$

$Q(y)$ is a double log-normal distribution (because of two $\mu$ and two $\sigma$ ) Fig.1, where $y$ represents the closing price in percentage; with respect to the variable $\ln (y), \mu_{p}$ is the mean of stop-profit price and $\mu_{l}$ is that of stop-loss one, $\sigma_{p}$ is the standard variation of stop-profit price and $\sigma_{l}$ is that of stop-loss one; the coefficient $c_{p}$ is the win ratio and $c_{l}$ is the loss one on the 
condition of $c_{p}+c_{l}=1 ; E(Y)$ is the expected (mean) value of $Y$, and $R S D(Y)=S D(Y) / E(Y)$ is the relative standard deviation of $Y, E_{p}=E\left(Y_{p}\right)$ and $E_{l}=E\left(Y_{l}\right)$. In practice, it is possible that traders close positions at $g$ (i.e. $y=0$ ) but the probability will be very low. Thus for simplification, we let $Q(y=0)=0$.

Statistically, in any periods we suppose investors of the same type (represented by lot sizes) have the same parameters of $Q(y)$; and the net win ratio $\left(c_{p}-c_{l}\right)$ is the same for all investors since it is mainly determined by the market characteristics. Moreover, it is impossible that $y<$ $-100 \%$ in practice. However, since most of transactions will be executed at the prices of $\ln (y)$ around $\mu$ when $\sigma$ is limited, the log-normal distribution of stop setting is an approximation to the reality. Similarly, its symmetry only works in the "local" range (not too large $Q$ ) as well.

In addition, let us show the relationship between the expected position time (duration of holding one position) $E_{t}(y)$ and the lot size $x$. Suppose the price process is geometric Brownian motion. Denote the current price as $S(0)$, the price at time $t$ as $S(t), S(t) / S(0)$ as $z=1$ $+y(y>0)$, the expected return as $\mu$ and the variance as $\sigma^{2}$. By Itô's lemma or binomial model, we have $\ln z \sim \Phi\left[\left(\mu-\sigma^{2} / 2\right) t, \sigma^{2} t\right]$. Thus for a certain $t$, its probability density at $\ln z$ is

$$
f_{t}(\ln z)=\frac{1}{\sqrt{2 \pi \sigma^{2} t}} \exp \left[-\frac{\left(\ln z-\left(\mu-\frac{\sigma^{2}}{2}\right) t\right)^{2}}{2 \sigma^{2} t}\right] .
$$

By swaping $t$ and $\ln z$ we get a function as $f_{\operatorname{lnz}}(t)$ that is a homeomorphic map of $F_{\operatorname{lnz}}(t)$ as the time PDF of $\ln z$ with respect to $t$. Though the distribution type of $F_{\ln z}(t)$ is unknown, we can show its topological characteristics through $f_{\ln z}(t)$ : since the probability measure is always equal to 1 , thus for a certain $t$, if $f_{l n z 1}(t)>f_{l n z 2}(t)$, then $F_{l n z 1}(t)>F_{l n z 2}(t)$, and vice versa (also true for "="), i.e. the relationship between two functions is unchangeable. By the mapping, for $y>0$ and $z_{1}>z_{2}$, it can be derived from (II.2.1) that when $t \rightarrow 0$ or $t \rightarrow+\infty, F_{\operatorname{lnz}}(t) \rightarrow 0 ; F_{\operatorname{lnz} 1}(t)$ and $F_{\operatorname{lnz} 2}(t)$ have only one point of intersection at $t_{0}=\left(\ln z_{1}+\ln z_{2}\right) /\left(2 \mu-\sigma^{2}\right) ; F_{\operatorname{lnz} 1}(t)$ has a mode at $t_{1}=\ln z_{1} /\left(\mu-\sigma^{2} / 2\right)$ and $F_{\operatorname{lnz} 1}(t)$ has one at $t_{2}=\ln z_{2} /\left(\mu-\sigma^{2} / 2\right)$ with $\operatorname{Mode}\left(F_{\operatorname{lnz} 1}\right)<\operatorname{Mode}\left(F_{\operatorname{lnz} 2}\right)$.

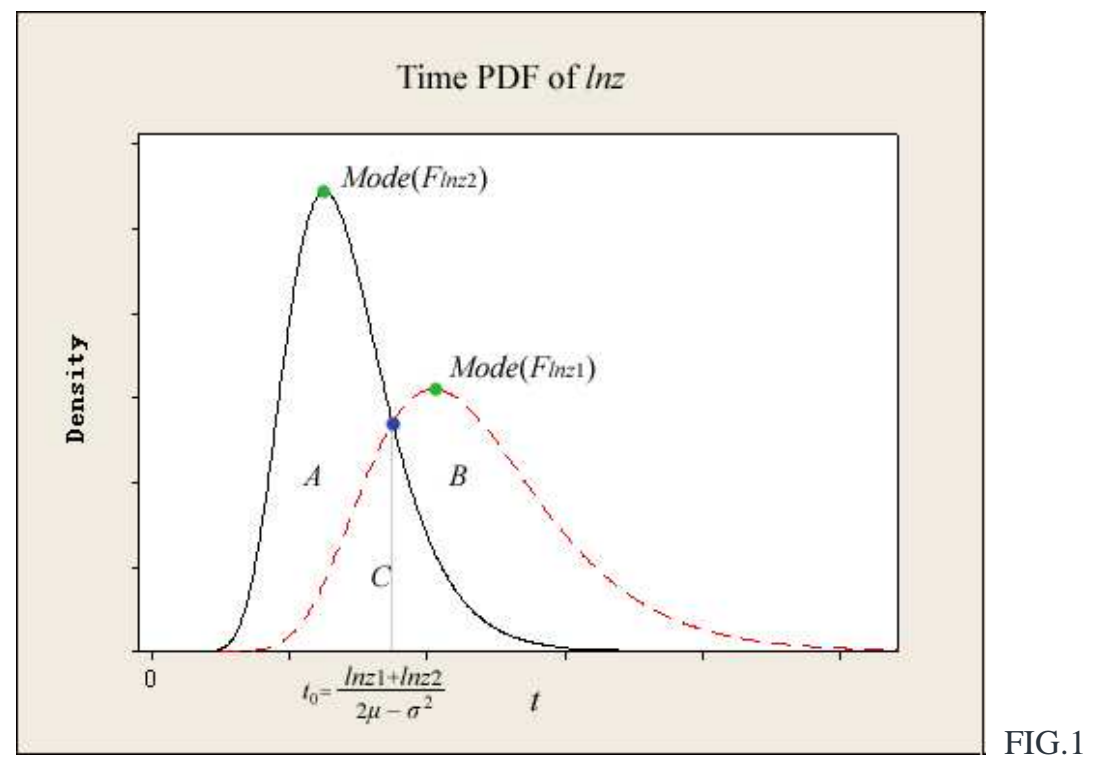


Suppose the expected time of closing positions at $z$

$$
\bar{t}^{-}(z)=\int_{0}^{+\infty} t \cdot F_{l n z}(t) \mathrm{d} t
$$

is absolutely convergent. (II.2.2) is equivalent to calculating the $\mathrm{X}$-axis bary centre of the area under $F_{l n z}(t)$. Denote three areas in Fig. 1 as

$$
\begin{gathered}
A=\int_{0}^{t_{0}} F_{\ln z_{2}}(t) \mathrm{d} t-\int_{0}^{t_{0}} F_{\ln z_{1}}(t) \mathrm{d} t \\
B=\int_{t_{0}}^{+\infty} F_{\ln z_{1}}(t) \mathrm{d} t-\int_{t_{0}}^{+\infty} F_{\ln z_{2}}(t) \mathrm{d} t \\
C=\int_{0}^{t_{0}} F_{\ln z_{1}}(t) \mathrm{d} t+\int_{t_{0}}^{+\infty} F_{\ln z_{2}}(t) \mathrm{d} t
\end{gathered}
$$

In addition to the common area $C$, the X-axis bary centre of area $A$ of $F_{\operatorname{lnz} 2}(t)$ must be lower than that of area $B$ of $F_{\operatorname{lnz} 1}(t)$ since they are located respectively on the left and right side of $t_{0}$, thus we have $t^{-}\left(z_{1}\right)>t^{-}\left(z_{2}\right)$ when $z_{1}>z_{2}$. In other words, $t^{-}(z) \propto z$ or $t^{-}(y) \propto y$ when $y>0$; similarly, $t^{-}(y) \propto|y|$ when $y<0$, which is a market characteristic independent of traders' probability density of closing positions at $y$. Hence, the expected position time of a log-normal distribution as $Q(y)$

$$
E_{t}(Y)=\int_{0}^{+\infty} t_{p}^{-}(y) Q(y) \mathrm{d} y+\int_{-\infty}^{0} t_{l}^{-}(y) Q(y) \mathrm{d} y
$$

Since

$$
E\left(Y_{p}\right)=\int_{0}^{+\infty} y Q(y) \mathrm{d} y, E\left(Y_{l}\right)=\int_{-\infty}^{0} y Q(y) \mathrm{d} y
$$

if $E\left(Y_{p}\right)>E\left(Y_{p}\right)^{\prime}$ and $\left|E\left(Y_{l}\right)\right|>\left|E\left(Y_{l}\right)^{\prime}\right|$, then $E_{t}(Y)>E_{t}(Y)^{\prime}$.

Theorem 1. (Position Time Theorem) if assumption one holds, then the expected position time of larger investors is longer than that of smaller ones under the condition that the distribution of security prices or its returns is log-normal.

As we have shown in chapter I, empirical data shows such distribution has long or fat tails. However, Position Time Theorem still roughly holds because the assumption of normality of price changes is the best approximation to the real market in most cases. The trick is, we do not directly apply the normality of price changes, instead, we employ the normality of stop setting to avoid the former's flaws. Of course, the theorem is still an approximation - stop size may not be rigorously monotone increasing, e.g. a few long-term investors may be small 
ones. But we focus on eigen behaviors ${ }^{33}$ rather than some local exceptions. In contrast, fat tail is a global problem and may lead to huge losses for all investors. For the same reason, in VSA it is not nessceary to seek Nash equilibriums or take interactive revisions into account, so we do not concern the interaction among personal behaviors though it is a major topic in many auction researches ${ }^{34}$, which is not for simplification but for actualization. For example, such problems as whether call auction is open or close do not matter in VSA. Hence, we only consider the trading rules in proofs. Then we have to show that the distribution of lot sizes is not completely random and has a strong impact on the market trend. The proofs will be made step by step as the following in chapter III:

1. Introducing the "volume spectrum" and showing some characteristics of it.

2. Proving the variety of volume spectrum does affect the expected return in call auction.

3. Proving continuous trading is "linearly equivalent" to call auction.

In addition, a demanding problem should be addressed: a large order is usually split into many small ones to reduce the impact cost, for example - such algorithm trading as Volume Weighted Average Price (VWAP), which may significantly distorts the distribution of lot sizes. An approach to recovering order splitting will be presented in the next chapter.

\section{The model of volume spectrum analysis}

\section{A. Volume Spectrum}

For a time window of trading, there are two kinds of filled orders: buy and sell orders, any one of them can be transformed to a "volume spectrum" as that of a sound. But unlike Fourier Transform, it features in a specific characteristic of scale as map. By analogy with Gutenberg-Richter Law, the magnitude of lot sizes can be measured by classifications of different amounts of partitions as the following procedure, which is called Half-scale Method (HSM).

For some collection of buy or sell orders in a time window, we suppose the maximum number of partitions $N_{0}=2^{k}$, where $k \in \mathbf{N}^{*}$. Then we design an initial division $d=d(m)$, a monotone decreasing function, where $m \in\left[1,2, \ldots, N_{0}\right]$. The $m$ th partition has orders of lot size $L$ as $d(m)$ $\geq L>d(m+1), d\left(N_{0}+1\right)=0$. Then merging each pair of adjacent partitions gives us a new division whose partition number $N_{1}=N_{0} / 2$, a half scale of the initial one - repeating the procedure covers half scales $N_{i}, i \in[1,2, \ldots, k]$. For the divisions of scales between $N_{0}$ and $N_{1}$, we design a series of local division functions $D=D(N, n)$, where $N \in\left[N_{1}+1, N_{1}+2, \ldots, N_{0}-1\right]$ and $n \in[1,2, \ldots, N]$, also a monotone decreasing function with respect to $n$ when $N$ is fixed. Then the divisions of scales between $N_{1}$ and $N_{2}$ can be made by merging each pair of adjacent partitions of divisions with even $N$ - repeating the procedure covers scales between $N_{i-1}$ and $N_{i}$. Hence, all scales from $N_{0}$ to 1 is traversed - one of reasons for making local divisions is due

\footnotetext{
${ }^{33}$ Luo, Is the Antitrust Policy Trustful? (I) - Strategy Mechanism of Lockefeller's Monopoly, pp.6-7.

${ }^{34}$ Myerson, Optimal Auction Design, pp. 58-73.
} 
to the difficulty of designing an overall division function $f(N, n)$ that fits the full range, another reason is showed in succedent proofs. For each partition of some scale, adding its orders on Y-axis gives us Single Volume Spectrum (SVS) as Fig.2.

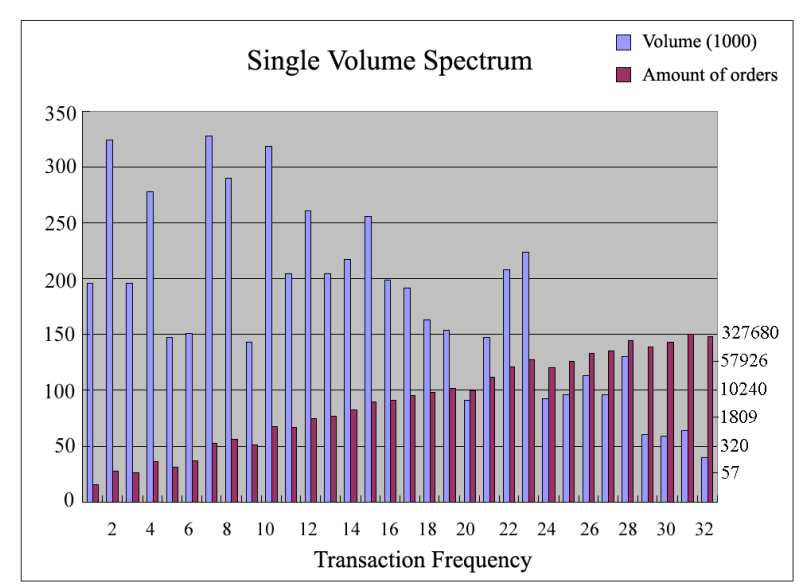

Fig.2

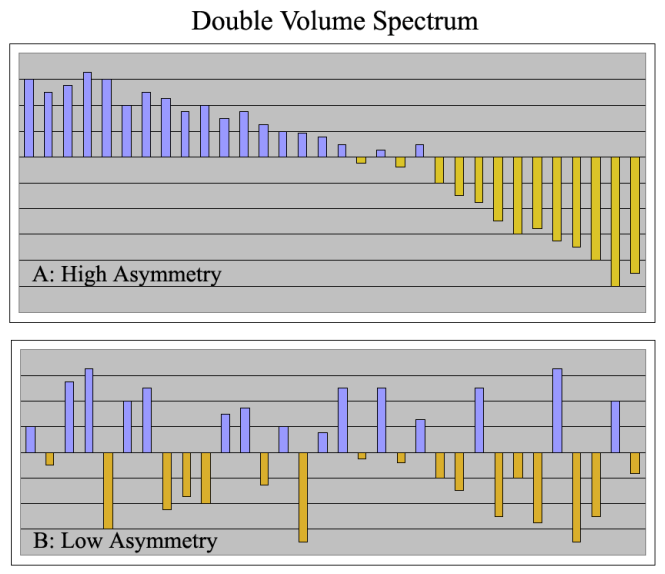

Fig.3

Generally speaking, the smaller the lot size, the larger the amount of transactions, i.e. the higher frequency of trading. Thus in Fig.2, for a time window $W, \mathrm{X}$-axis represents the transaction frequency and the $\mathrm{Y}$-axis is the volume intensity. Therefore, the volume spectrum could be likened to the sound spectrum. From the symmetry of stop range in Assumption 1, it follows that both sides of orders have the same magnitude of effect on the future price. On some scale, let its SVS of sell orders be subtracted from that of buy orders, we get a Double Volume Spectrum (DVS) as Fig.3. Then the sum of blue columns (positive in this case) must be equal to that of yellow ones (negative in this case) to cancel each other, its absolute value is called Trade Volume Difference (TVD), denoted as $\delta$.

The distribution of DVS and its TVD are subject to the division function and especially parameter $N$. We start the division at a certain large $N_{0}$ defined as before, so that each partition only contain orders with the exactly same lot size or nothing. Thus, only a little buy and sell orders cancel each other, and $\delta_{0}$ is close to the total volume $V$. Thus DVS $\mathrm{S}_{0}$ is almost random distributed, and its contour has a large amount of zero points $Z$, its amount is denoted as $M_{0}$. Then we take $N_{1}=N_{0} / 2$ and then $\delta_{1} \leq \delta_{0}, M_{1} \leq M_{0}$, since it is quite possible that there is a zero point between any two adjacent partitions - thus more two-side lots may cancel each other. Then repeating the HSM procedure of $N_{i}=N_{i-1} / 2$ until $N_{k}=1$, gives us $\delta_{0} \geq \delta_{1} \geq \delta_{2} \ldots \geq$ $\delta_{i}$ and $M_{0} \geq M_{1} \geq M_{2} \ldots \geq M_{i}$, i.e., as $N$ falls, normally $\delta$ and $M$ also fall. By the way, it may not be strictly monotone decreasing in the interval $\left[N_{i-1}, N_{i}\right]$, but the inequality holds for any series of half scale. Let $r_{i}=\operatorname{round}\left(N_{0} / N_{i}\right)$, then $S_{i}=1: r_{i}$ is defined as the $i$ th scale of DVS.

However, before $N_{k}=1$ the Assumption 2 assures a $N_{c}$ that when $N_{i}=N_{c}$ only one zero point remains. When $N_{i}<N_{c}$, TVD gets to stabilize with slight fluctuation, i.e. a stable phase. If no stable phase is reached until $N$ goes to 1 , the ergodic result will conflict with the assumption that $q(x)$ is statistically monotone, which is derived from the fact that the sign of each column in DVS is the same as that of $\left(2 q^{\mathrm{s}}-1\right)$, where $q^{\mathrm{s}}$ is the weighted average $q$ of all orders in this partition, and its volume 


$$
V^{S}=\left(2 q^{S}-1\right)\left(\sum_{i=1}^{n} x_{i}+\sum_{j=1}^{m} y_{j}\right), \text { where } x_{i} \text { is buy order and } y_{j} \text { is sell one. }
$$

The DVS, TVD, zero point and scale of $N_{c}$ are respectively called critical DVS (DVS ${ }^{\mathrm{C}}$ or $\omega_{c}$ ), critical TVD $\left(\mathrm{TVD}^{\mathrm{C}}\right.$ or $\left.\delta_{c}\right)$, critical zero point $\left(Z_{c}\right)$ and critical scale $\left(S_{c}\right) . \delta_{c}$ could be positive or negative, whose sign is subject to those columns to the left of $Z_{c}$.

Let the total volume be $V$ and that subtracted by the volume of high frequency trading (HFT) be $V^{a d j}$, Information Asymmetry Degree (IAD) is defined as $H=\left(\left|D_{c}\right| / V\right) \cdot 100 \%$ or $H^{\text {adj }}=$ $\left(\left|D_{c}\right| / V^{a d j}\right) \cdot 100 \%$ that represents the amount of information. It implies the effectiveness of spectrum analysis. Fig.3.A is a possible example of asymmetric information in reality, when $N$ is close to $N_{c}$. In contrast, if lot sizes (or probability q) are uniformly distributed at any scale level of DVS, then no $N_{c}$ will appear in the ergodic process from $N_{0}$ to 1 , or in reality $N_{c}$ and $\delta_{c}$ is quite small, which means $H \approx 0$, and $\mathrm{DVS}^{\mathrm{C}}$ has only a few short columns, thus spectrum analysis is almost impossible (Fig.3.B, at a status of $N_{i}>N_{c}$ ).

We can test Assumption 2 with actual trading data to see if IAD is large enough statistically. Actually Assumption 1 and 2 are related each other - if the information asymmetry is remarkable at the macro-level, it will imply the reality of Assumption 2 at the micro-level. On the other hand, it is hard to test assumptions of other finance theories - a typical example is CAPM. As Richard Roll's critique, the market portfolio is unobservable since it is impossible to include all possible available assets, thus the assumption of mean-variance efficiency of the portfolio is untestable and a tautology of CAPM equation. ${ }^{35}$

As for HFT, no matter what strategies they use, HFT players actually replicate the role of market maker, i.e. the middleman between buyers and sellers. Not to carry unhedged positions overnight, they must try to balance the sizes of two-sided orders in every twin transaction as possible. Thus, most orders of HFT (or day trading) will cancel each other in the DVS, i.e. the effect of HFT could be almost eliminated.

The problem of recovering order splitting could be solved in the framework of volume spectrum as well. Though many traders adopt VWAP algorithms, it is almost impossible to recover split orders in time series data because it features not quasi-isometry of time interval but that of lot size, which means in VWAP or other similar algorithms the lot sizes of orders are relatively close since the volume keeps stable during a relatively short time window of trading, except for the opening and closing time in which such algorithms are unnecessary. As the ergodic process of scale in a SVS runs, such orders accumulate and then highlight at certain partitions (its volume column will be much higher than the adjacent ones) on some scale, which is supposed to occur before the critical scale - the closer the lot sizes of such orders, the earier (i.e. on the larger scale) the highlighted partitions appear.

For a simplest example, denote the volume of a highlighted partition as $V_{h}$, and those of adjacent partitions as $V_{1}$ and $V_{2}$, thus the estimated lots of original order

$$
L_{0}=V_{h}-\left(V_{1}+V_{2}\right) / 2
$$

\footnotetext{
35 Roll, Richard (March 1977). "A critique of the asset pricing theory’s test Part I: On past and potential testability of the theory", Journal of Financial Economics.
} 
Then we move the recovered order to a new partition according to $L_{0}$, and remove the splitting orders in the previous partition (a recognition algorithm needed), and then continue the ergodic process. But before that, $V_{h}$ should be compared with that of the corresponding partition in another SVS - if they are significantly close, it will be quite possible that they belong to HFT (or day trading). Thus, HFT volume could be estimated to adjust IAD.

\section{B. Proof in Call Auction}

It is supposed that every trader makes a stop setting with a PDF as $Q(y)$ at the time of entry. For a time window $W$, assume there is a trade $G$ with $\omega_{c}$ and $\delta_{c}$, in which some positions are opened. Then if a new auction is called, every one of traders holding these positions will estimate a possible trading price $p_{e}$ that varies from trader to trader. We only consider a local range of $p_{e} \in[\alpha, \beta]$ that is a bit larger than the possible range of filled orders' ask and bid prices. Within this range, not all but some of traders will place their closing orders at random prices with the probability

$$
\operatorname{Pr}_{x}=r \int_{\alpha}^{\beta} Q_{x}(y) \mathrm{d} y
$$

where $0<r<1$ is a ratio subject to the characteristics of market, it is equal for every trader in one auction. Then some of these orders are filled, which implies conversion positions that long and short ones switch each other (we define purchase as long and sale as short) in spite of those whose traders leave the stock for a relatively long time, since we assume that in general the proportions of these orders in ask and bid ones are very close except for some cases with extreme volatility. Of course traders will open new positions, which is called external condition as stochastic disturbance in price process.

Denote the opening price as $p$ and lots of order as $x$. In the initial auction, there are two binary sets $G_{s}$ and $G_{b}$ as $[p, x]$ of filled sell and buy orders. In the new auction, sort submitted sell orders in ascending order of prices to get a binary sequence: $S=\left[\left(S_{1}, T_{1}\right) ;\left(S_{2}, T_{2}\right) ; \ldots ;\left(S_{M}\right.\right.$, $\left.T_{M}\right)$ ], where $S_{1} \leq S_{2} \ldots \leq S_{M}, S_{j}$ is the ask price and $T_{j}$ is its lot size; and sort submitted buy orders in descending order of prices: $B=\left[\left(B_{1}, C_{1}\right) ;\left(B_{2}, C_{2}\right) ; \ldots ;\left(B_{N}, C_{N}\right)\right]$, where $B_{1} \geq B_{2} \ldots \geq$ $B_{N}, B_{i}$ is the bid price and $C_{i}$ is its lot size. Orders with the same ask or bid price are sorted by the time priority principle. Suppose the lowest matched bid is $B_{n}$ with $C_{n}-R$ lots, $R \in[0$, $\left.1,2, \ldots, C_{n}\right)$, i.e. $C_{n} \geq T_{m}$; and the highest matched ask is $S_{m}$ with $T_{m}$ lots; thus $B_{n} \geq S_{m}$. Let the sequence of filled sell orders $s=\left[\left(S_{1}, T_{1}\right) ;\left(S_{2}, T_{2}\right) ; \ldots ;\left(S_{m}, T_{m}\right)\right], s \in S$; and that of filled buy orders $b=\left[\left(B_{1}, C_{1}\right) ;\left(B_{2}, C_{2}\right) ; \ldots ;\left(B_{n}, C_{n}\right)\right], b \in B$. Thus, the trade will be executed at the price

$$
\begin{array}{r}
P=\left(B_{n}+S_{m}\right) / 2 \\
\text { and } \quad \sum_{i=1}^{n} C_{i}-R=\sum_{j=1}^{m} T_{j}
\end{array}
$$

Then for the same $W$, assume there is another trade $G^{\prime}$ with $\omega_{c}{ }^{\prime}$ and $\delta_{c}{ }^{\prime}$, but $G$ and $G$ ' have the same total volume and price distribution. Consider a simple example: $\omega_{c}$ ' comes from changing $\omega_{c}$ by shifting some volume in a partition to another one on the right side, which 
could be made in two ways: (1) some orders in $G_{b}$ are splitted into many smaller ones in $G_{b}$ ' or (2) some orders in $G_{s}$ are merged into a few larger ones in $G_{s}$, , but all with the same opening price $p_{0}$. For case (1), denote the distribution of closing prices of such one small order in $G_{b}$ ' as $(Z)$ ', and its same portion of the corresponding large order in $G_{b}$ as $(Z)$. Thus for case (1), $E(Z)^{\prime}<E(Z)$ from Assumption One. For the range of $p_{e}$, there are two situations: (a) $[\alpha, \beta] \in \mathbf{R}+$, (b) $[\alpha, \beta] \in \mathbf{R}$-. And for case (1.a), there are three possibilities.

Case (1.a.1). Suppose $R S D(z)^{\prime}>R S D(z)$, then $\sigma^{\prime}>\sigma$ from (II.1.2), $\mu^{\prime}<\mu$ from (II.1.3) because we alreadly have $E(Z)^{\prime}<E(Z)$. Then from (II.1.1) there are two points $a$ at $z_{1}$ and $b$ at $z_{2}$ of intersection of $Q(z)^{\prime}$ and $Q(z)$ as the solutions of

$$
\frac{\left(\ln z-\mu^{\prime}\right)^{2}}{\sigma^{\prime^{2}}}-\frac{(\ln z-\mu)^{2}}{\sigma^{2}}=\ln \left(\frac{\sigma^{2}}{\sigma^{\prime 2}}\right)
$$

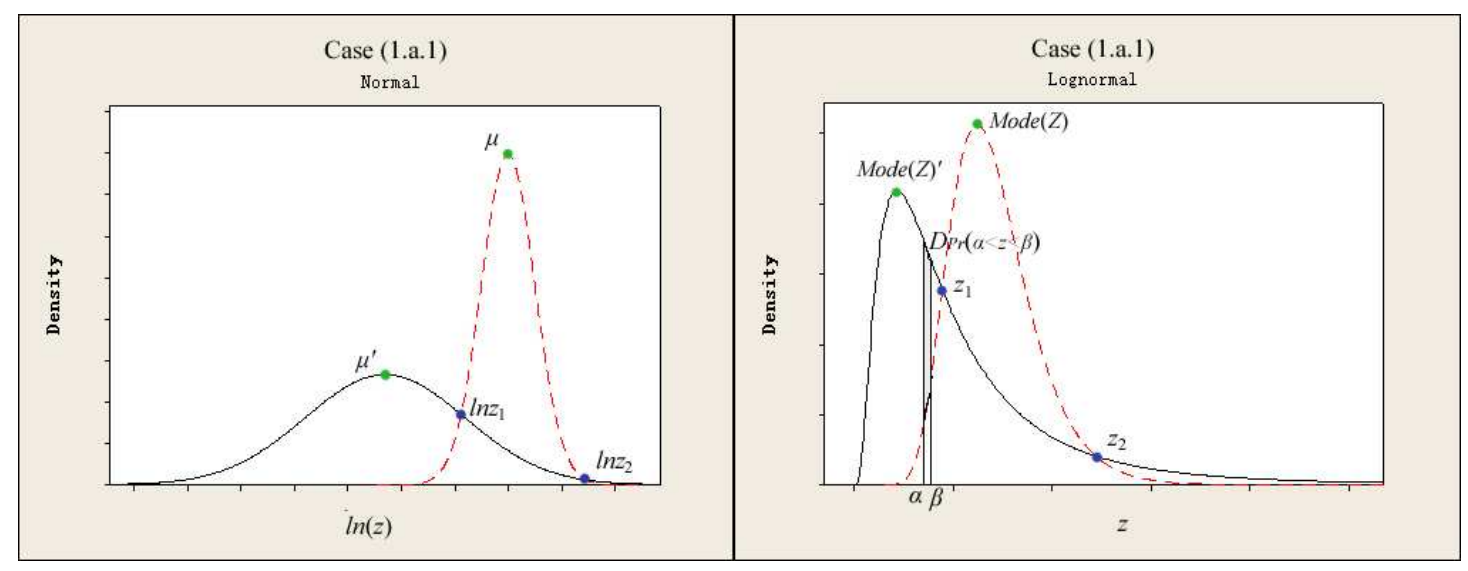

FIG.4

Hence, if $\beta \leq z_{1}$, then

$$
\int_{\alpha}^{\beta} Q\left(z, \mu^{\prime}, \sigma^{\prime}\right) \mathrm{d} z>\int_{\alpha}^{\beta} Q(z, \mu, \sigma) \mathrm{d} z, \beta \leq z_{1}
$$

i.e. $\operatorname{Pr}(Z)^{\prime}>\operatorname{Pr}(Z)$ for the probability of closing position at $[\alpha, \beta]$; and if $\alpha \geq z_{1}$ and $\beta \leq z_{2}$, then

$$
\int_{\alpha}^{\beta} Q\left(z, \mu^{\prime}, \sigma^{\prime}\right) \mathrm{d} z<\int_{\alpha}^{\beta} Q(z, \mu, \sigma) \mathrm{d} z, \alpha \geq z_{1}, \beta \leq z_{2}
$$

i.e. an opposite impact.. Thus by the Position Time Theorem, in the short term (Z)' has stronger impact on the stock price than $(Z)$; but in the medium term, the impact is reversed; and in the long time $\left(\alpha \geq z_{2}\right)$, both impacts are very weak and can be ignored (Fig.4). Denote the difference between $E(Z)^{\prime}$ and $E(Z), \operatorname{Pr}(Z)^{\prime}$ and $\operatorname{Pr}(Z), R S D(Z)^{\prime}$ and $R S D(Z)$, respectively as $D_{E}, D_{P r}$, and $D_{R}$. Then it can be proved that $D_{P r} \propto D_{E}$ for a certain range of $[\alpha, \beta]$, when $D_{R}$ is constant. Repeat the reasoning for any one of such orders in case (1), we can add all these volume-weighted probabilities.

Therefore, more sell orders may be asked in $G$ ' when the external condition do not change. If some bid prices of these orders are lower than $P$ in $G_{b}$, for holding equation (III.1.1) must $m$ ' 
$\leq m$ and $n^{\prime} \geq n$ according to the maximum volume principle. Then $S^{\prime}{ }_{m} \leq S_{m}$ and $B^{\prime}{ }_{n} \leq B_{n}$, and finally $P^{\prime} \leq P$ because of equation (III.1.1). It is still possible that in subsequent auctions $P_{1}{ }^{\prime} \leq$ $P_{1}, P_{2}{ }^{\prime} \leq P_{2}, \ldots$ until the inequality reverses in the medium term. In other words, in any $k$ th auction of the short term, the expected return $R_{k}{ }^{\prime} \leq R_{k}$, but in the medium term, $R_{k}{ }^{\prime} \geq R_{k}$. For case (2.a.1), the situation is symmetric - less buy orders may be bid in $G^{\prime}$, thus one can get the same result. Then let us build a few lemmas as preparations for the next proof.

Lemma 1. For two normal PDFs $A(x)$ with $\mu_{1}$ and $\sigma_{1}^{2}, B(x)$ with $\mu_{2}$ and $\sigma_{2}^{2}$, if $\mu_{1} \neq \mu_{2}$ and $\sigma_{1} \neq$ $\sigma_{2}$, there are two and only two points of intersection $\in(-\infty,+\infty)$; else if $\mu_{1} \neq \mu_{2}$ and $\sigma_{1}=\sigma_{2}$, only one point of intersect appears at $x=\left(\mu_{1}+\mu_{2}\right) / 2$, and another one presents at infinity. It follows directly from (III.2.1).

Lemma 2. For a log-normal PDF $Q($ lny $)$ with $\mu$ and $\sigma^{2}$, suppose $\sigma$ increases to $\sigma^{\prime}$, which makes a new PDF as $Q(y)^{\prime}$. Their normal PDFs have two points of intersection at $\ln y_{1}$ and $\ln y_{2}, \ln y_{1}<\ln y_{2}$. Thus for any $\ln y \in\left(\ln y_{1}, \ln y_{2}\right), Q(\ln y)^{\prime}<Q(\ln y)$; and for any $\ln y<\ln y_{1}$ or $\ln y$ $>\ln y_{2}, Q(\ln y) '>Q(\ln y)$. It follows from Lemma 1 and (II.1.1). When $\sigma$ decreases, vice versa.

Lemma 3. For two normal PDFs as Lemma 1, if $\mu_{1}>\mu_{2}$ and $\sigma_{1}<\sigma_{2}$, then both points $x_{1}$ and $x_{2}$ of intersection locate on the right arm of $B(x)$ with $\mu_{2}<x_{1}<\mu_{1}<x_{2}$. It follows from Lemma 2, (II.1.2) and (II.1.3).

Let us prove an ergodic process by fixing $E(Z)$ and increasing $R S D(Z)$. Thus $\sigma$ increase and $\mu$ decrease from (II.1.2) and (II.1.3). During the time, intersection point $a$ moves up to the left along the right arm of $Q(z)^{\prime}$ and $b$ moves down to the right. Then $R S D(Z)$ continues to increase until $\sigma^{\prime}=\sigma$, from Lemma 1 it follows that $\ln z_{1}=\left(\mu^{\prime}+\mu\right) / 2$, and $b$ disappears (presents) at infinity. When $\operatorname{RSD}(Z)$ continues to increase, point $b$ will come forth again on the left arm of $Q(z)^{\prime}$ and get to move up to the right. Then let $R S D(Z)$ continue to increase until $\ln z_{1}=\mu$ (denote as $\mu_{m}$ ), this point of intersection is the highest one on the right arm of $Q(z)$ '.

Proof. Substituting $\ln z_{1}=\mu_{m}$ or $\ln z_{2}=\mu_{n}$ into (III.2.1) gives us:

$$
\mu_{m}=\mu^{\prime}+\sigma^{\prime} \sqrt{\ln \left(\frac{\sigma_{m}^{2}}{\sigma^{\prime 2}}\right)}, \mu_{n}=\mu^{\prime}-\sigma^{\prime} \sqrt{\ln \left(\frac{\sigma_{n}^{2}}{\sigma^{\prime 2}}\right)}
$$

and with

$$
\mu_{m}=\ln E(Z)-\frac{1}{2} \sigma_{m}^{2}, \mu_{n}=\ln E(Z)-\frac{1}{2} \sigma_{n}^{2}
$$

then

$$
\begin{aligned}
& \frac{1}{2} \sigma_{m}^{2}+\sqrt{\sigma^{\prime 2} \ln \left(\frac{\sigma_{m}^{2}}{\sigma^{\prime 2}}\right)}+\mu^{\prime}-\ln E(Z)=0 \\
& \frac{1}{2} \sigma_{n}^{2}-\sqrt{\sigma^{\prime 2} \ln \left(\frac{\sigma_{n}^{2}}{\sigma^{\prime 2}}\right)}+\mu^{\prime}-\ln E(Z)=0
\end{aligned}
$$


Thus $\sigma_{m}, \mu_{m}$ and $\sigma_{n}, \mu_{n}$ can be determined by (III.2.2). If $\ln z_{1}<\mu_{m}$, then $Q\left(\ln z_{1}\right)^{\prime}>Q\left(\mu_{m}\right)$ ' because $Q(z)^{\prime}$ is monotone decreasing on its right arm. Since $\mu_{m}$ is the global maximum of its PDF $Q_{m}$, thus by the symmetry of normal distribution, for any $\ln z Q(\ln z)>Q_{m}(\ln z)$, i.e. $\int Q>$ $\int Q_{m}$ that is in contradiction with $\int Q=\int Q_{m}=c_{p}$ from (II.1.1). Hence, $\ln z_{1} \geq \mu_{m}$ must hold for the whole ergodic process, which means the point of intersection with $\ln z_{1}=\mu_{m}$ is the highest one on the right arm of $Q(z)^{\prime}$. It is the same reasoning to prove the point with $\ln z_{2}=\mu_{n}$ is the highest one on the left arm of $Q(z)$ '. From Lemma 3, at the beginning (case (1.a.1)) we have $\mu_{m}<\ln z_{1}<\mu<\ln z_{2}$, and $Q\left(\ln z_{2}\right)<Q\left(\ln z_{1}\right)<Q\left(\mu_{m}\right)<Q(\mu)$. And then let us make a new PDF as $Q(z)$ " with $\sigma_{m}>>\sigma$ " $>\sigma$, thus the span between two poins of intersection of $Q(z)$ "' and $Q(z)$ is so small that $\ln z_{1}$ and $\ln z_{2}$ are out of it. From Lemma 2, points around and including them move up. Hence, point $a$ moves up to the left and point $b$ moves down to the right. It is similar to prove that after point $b$ comes forth again on the left arm of $Q(z)$ ' it will get to move up to the right.

Case (1.a.2). In this case (Fig.4), $\sigma_{m} \leq \sigma<\sigma^{\prime}$. Thus when $z<z_{2}, Q(z)^{\prime}<Q(z)$ ); when $z_{2}<z<z_{1}$, $Q(z)^{\prime}>Q(z)$; and when $z>z_{1}, Q(z)^{\prime}<Q(z)$. Therefore, in the very short term $\operatorname{Pr}(Z)^{\prime}<\operatorname{Pr}(Z)$ but with very low difference, which can be ignored; in the short term $\operatorname{Pr}(Z)^{\prime}>\operatorname{Pr}(Z)$ and in the medium term $\operatorname{Pr}(Z)^{\prime}<\operatorname{Pr}(Z)$, which has some comparability with case(1.a.1) (Fig.6). It is possible that this case will happen, but not as frequently as case(1.a.1) because larger investors often have the advantage to get lower $R S D$. Moreover, its $D_{P r}$ is lower than that of case (1.a.1) according to the above argument, i.e. its impact on the stock price is weaker.

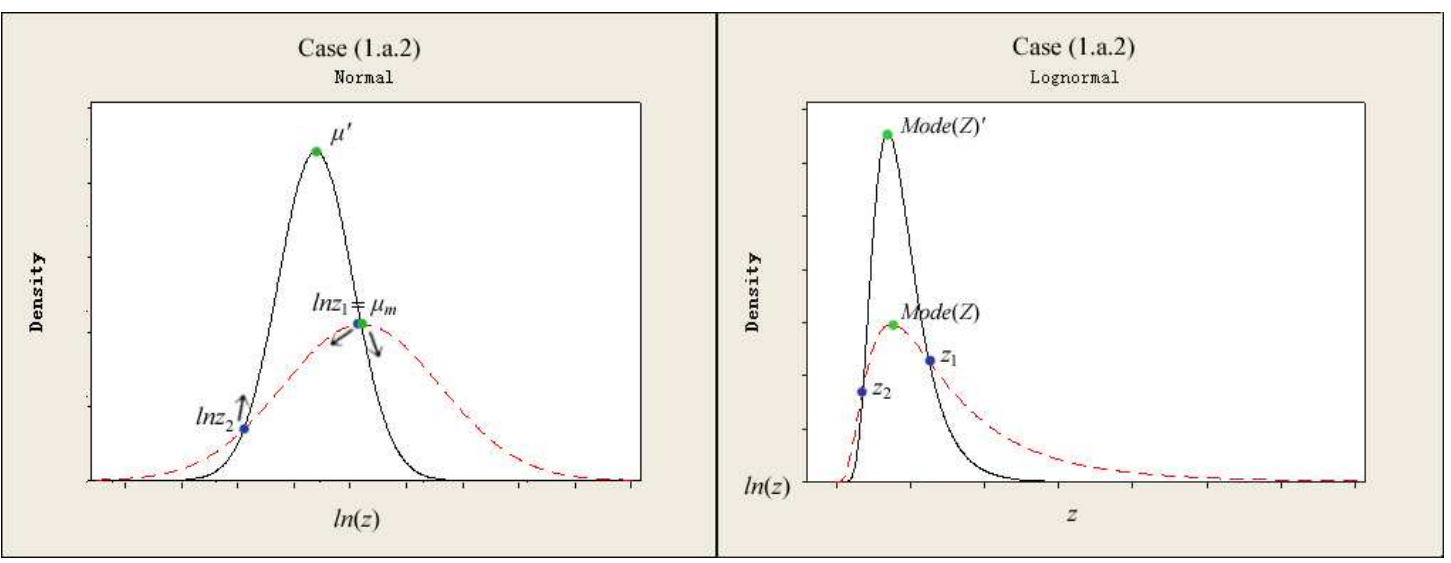

FIG.5

Case (1.a.3). Let $R S D(z)$ continue to increase so that $R S D(z)^{\prime}<<R S D(z)$ until $\ln z_{2}=\mu$ (denote as $\mu_{n}$ ), this point of intersection is the highest one on the left arm of $Q(z)^{\prime}$. During the time, point $b$ moves up to the right on the left arm of $Q(z)$ ' and point $a$ moves down to the right on the left arm of $Q(z)$. The reasoning is similar to that of case (1.a.2). In this case $\sigma_{n} \leq \sigma<\sigma_{m}$. Thus when $z<z_{1}, Q(z)^{\prime}<Q(z)$; when $z_{1}<z<z_{2}, Q(z)^{\prime}>Q(z)$; and when $z>z_{2}, Q(z)^{\prime}<Q(z)$. The impact of case (1.a.3) is much different from that of case (1.a.1): a sharp fluctation in the short term and then quickly calms down in the medium and long term. Actually, the two cases are almost inverse to each other (Fig.6). 


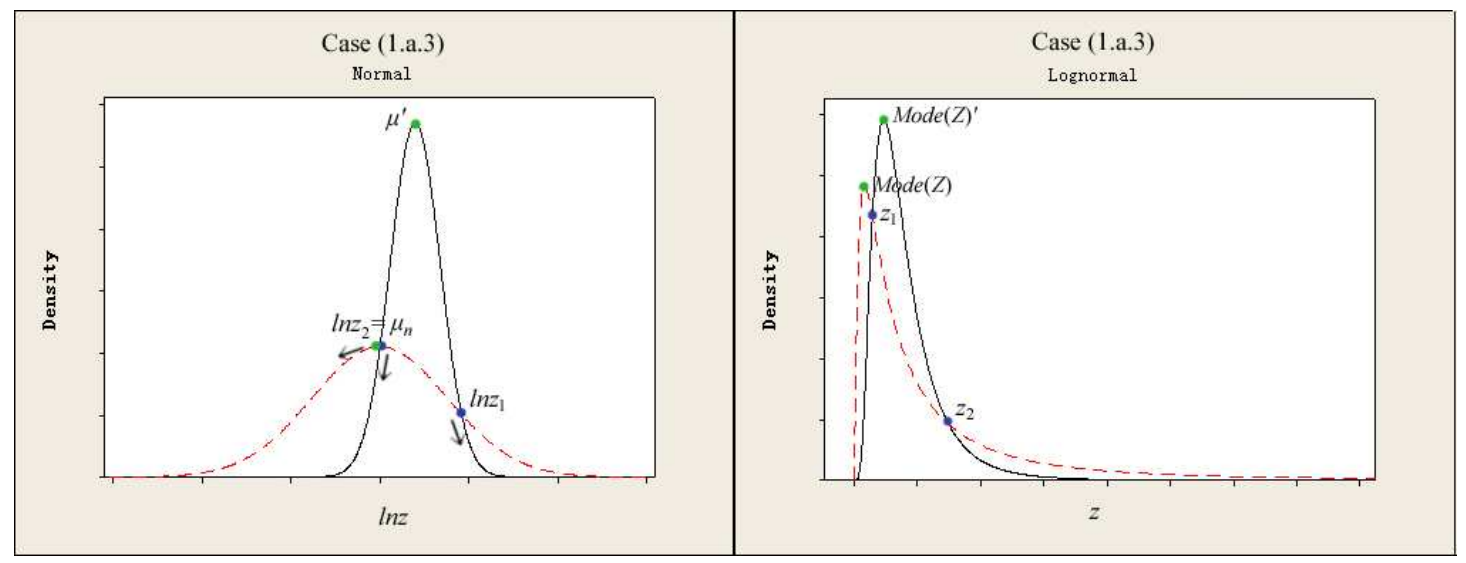

FIG.6

This case is almost impossible not only because of larger investors' advantage, and also because of its own characteristic - let us show the fact. Since in this case $\operatorname{RSD}(Z)>R S D(Z)$ ', consider an extreme situation: $R S D(Z)=100 \%$; and $R S D(Z)^{\prime}=0 \%$. Then from (II.1.3), $\mu<\mu$ ' give us

$$
\frac{E(Z)}{\sqrt{1+R S D^{2}(Z)}}<\frac{E(Z)^{\prime}}{\sqrt{1+R S D^{2}(Z)^{\prime}}}
$$

Since $\operatorname{RSD}(Z)=1$; and $\operatorname{RSD}(Z)^{\prime}=0$, then

$$
\frac{E(Z)}{E(Z)^{\prime}}<\sqrt{2}
$$

e.g. if $E(Z)^{\prime}=10 \%$, then $E(Z)<14.1 \%$, only a little difference even when the situation is almost impossible in practice. In fact, case (1.a.3) may occur only when $E(Z)$ ' and $E(Z)$ are very close, e.g. $R S D(Z)=1 / 3, R S D(Z)^{\prime}=1 / 10$, then $E(Z) / E(Z)^{\prime}<1.05$. Thus, their difference of impact is little and can be ignored.

In fact, these cases could be classified by $\sigma_{m}$ and $\sigma_{n}$ that are called critical $\sigma$. For any two PDFs as the above example, denote the initial $z_{1}$ as $z_{0}$. When $\ln z_{1}$ starts from $\ln z_{0}$ and goes to $\mu_{m}\left(\sigma \leq \sigma_{m}\right)$, it is feasible to analyze; then $\ln z_{2}$ goes to $\mu_{n}\left(\sigma_{m} \leq \sigma<\sigma_{n}\right)$, it gets to be hard to analyze; finally after $\ln z_{2}$ leaves from $\mu_{n}\left(\sigma \geq \sigma_{n}\right)$, it is almost impossible to analyze. We say that a case of two PDFs $Q(Z)$ and $Q(Z)^{\prime}$ is feasible to analyze iff $\sigma \leq \sigma_{m}$ is satisfied when $\sigma_{m}$ is subject to $E(Z), \mu^{\prime}$ and $\sigma^{\prime}$; or $R S D(Z)<R S D\left(Z_{m}\right)$ is satisfied when $R S D\left(Z_{m}\right)$ is subject to $E(Z)$, $E(Z)^{\prime}$ and $R S D(Z)^{\prime}$. Let us implement a numerical simulation to exploit the idea.

In fact, (III.2.2) can be rewritten by (II.1.2a) as

$$
\frac{1}{2}\left(\sigma_{m}^{2}-\sigma^{\prime 2}\right)+\sqrt{\sigma^{\prime 2} \ln \left(\frac{\sigma_{m}^{2}}{\sigma^{\prime 2}}\right)}-\ln \left(\frac{E(Z)}{E(Z)^{\prime}}\right)=0
$$




$$
\frac{1}{2}\left(\sigma_{n}^{2}-{\sigma^{\prime 2}}^{2}-\sqrt{\sigma^{2} \ln \left(\frac{\sigma_{n}^{2}}{\sigma^{\prime 2}}\right)}-\ln \left(\frac{E(Z)}{E(Z)^{\prime}}\right)=0\right.
$$

by (II.1.2), we can transform the independent variable $\sigma^{\prime}$ into $R S D(Z)^{\prime}$ ' and dependent variable $\sigma_{m}$ or $\sigma_{n}$ into $\operatorname{RSD}\left(Z_{m}\right)$ or $R S D\left(Z_{n}\right)$. It is observed that if $E(Z) / E(Z)$ ' is a constant, then (III.2.3) remains unchanged, which implies that $E(Z) / E(Z)$ ' can be treated as a single variable. Hence, we define the relative difference between $E(Z)$ and $E(Z)^{\prime}$ as

$$
D_{E}=\frac{E(Z)-E(Z)^{\prime}}{E(Z)^{\prime}} \times 100 \%
$$

For the case of $D_{E}=100 \%, \operatorname{RSD}(Z)^{\prime}=15 \%$ and $\operatorname{RSD}(Z)=30 \%$ are arbitrarily chosen, and refer its PDF image to Fig.4. From (II.1.2), (II.1.3), and (III.2.2), we get the following table by numerical solution (denote $M o d e=e^{\mu-\sigma^{\wedge} 2}$ as $M$ ):

\begin{tabular}{|c|c|c|c|c|c|c|c|c|c|c|}
\hline A. & \multicolumn{3}{|c|}{$Z$} & $Z$ & \multicolumn{3}{c|}{$Z_{m}$} & \multicolumn{3}{c|}{$Z_{n}$} \\
\hline$E$ & \multicolumn{3}{|c|}{$15 \%$} & \multicolumn{6}{c|}{$30 \%$} \\
\hline \multirow{2}{*}{$R S D$} & 1 & 2 & 3 & \multirow{2}{*}{$<\operatorname{RSD}\left(Z_{m}\right)$} & 1 & 2 & 3 & 1 & 2 & 3 \\
\cline { 2 - 11 } & $10 \%$ & $50 \%$ & $80 \%$ & & $127 \%$ & $94.1 \%$ & $116 \%$ & $231 \%$ & $472 \%$ & $696 \%$ \\
\hline \multirow{2}{*}{$\sigma$} & 0.100 & 0.472 & 0.703 & $<\sigma_{m}$ & 0.983 & 0.797 & 0.921 & 1.359 & 1.775 & 1.975 \\
\hline$\mu$ & -1.90 & -2.01 & -2.14 & $>\mu_{m}$ & -1.68 & -1.52 & -1.63 & -2.13 & -2.78 & -3.16 \\
\hline$M$ & $14.8 \%$ & $10.7 \%$ & $7.2 \%$ & $>M\left(Z_{m}\right)$ & $7.1 \%$ & $11.6 \%$ & $8.4 \%$ & $1.9 \%$ & $0.3 \%$ & $0.1 \%$ \\
\hline
\end{tabular}

And 2-demension function images of (III.2.3) for $D_{E}=20 \%, 50 \%$ and 100\% (Fig.7):
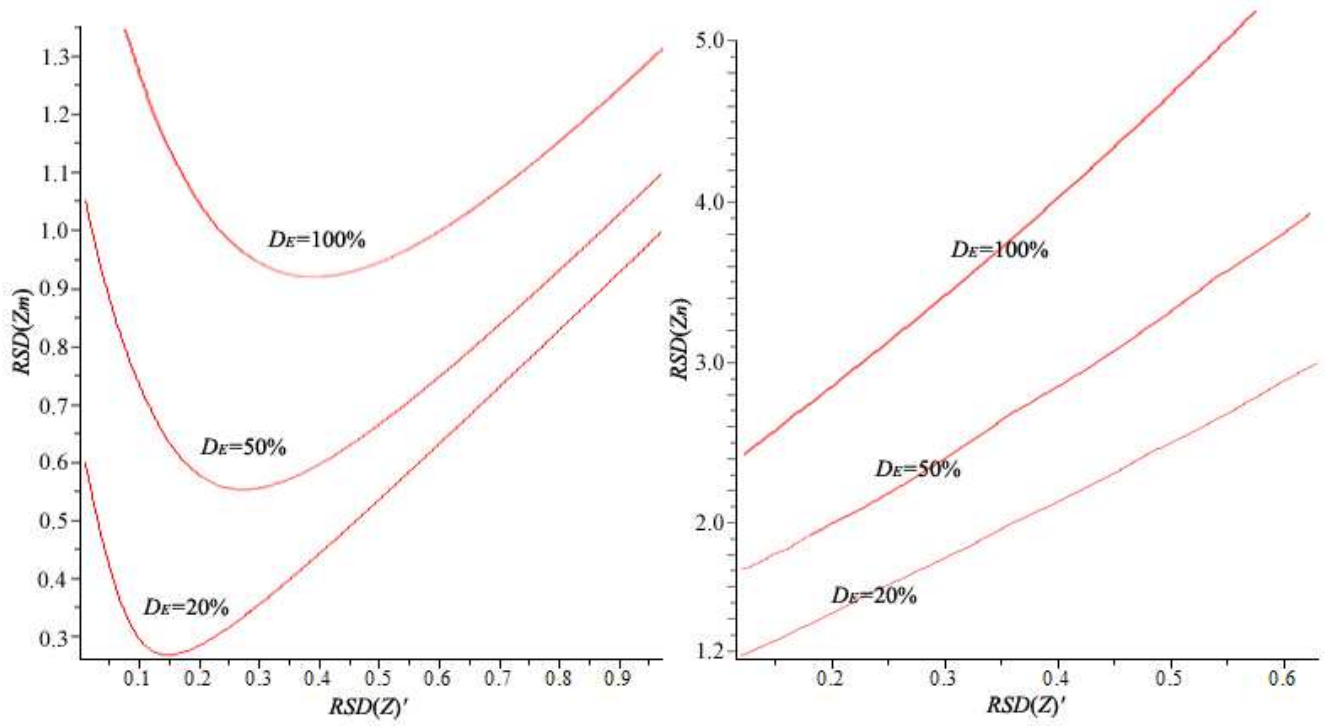

FIG.7

For $D_{E}=100 \%$, all $\operatorname{RSD}\left(Z_{m}\right)>92 \%$, the minimum at $R S D(Z)^{\prime} \approx 38 \%$. From the results of simulation, if $R S D>100 \%$, the trader will have considerable chances to close profit positions at very low or very high prices - it is quite impossible that there are many such traders at the 
same time or they have fat tails. Thus, it is easy to hold $R S D(Z)<R S D\left(Z_{m}\right)$ in cases of $D_{E}=$ $100 \%$ and our analysis are feasible. On the other hand, cases of $R S D(Z)>R S D\left(Z_{n}\right)$ are impossible. For $D_{E}=50 \%$, all $R S D\left(Z_{m}\right)>56 \%$, the minimum at $R S D(Z)^{\prime} \approx 27.5 \%$. For $D_{E}=$ $20 \%$. All $R S D\left(Z_{m}\right)>27 \%$, the minimum at $R S D(Z)^{\prime} \approx 15 \%$. We can see more cases in its $3 \mathrm{D}$ image (Fig.8):
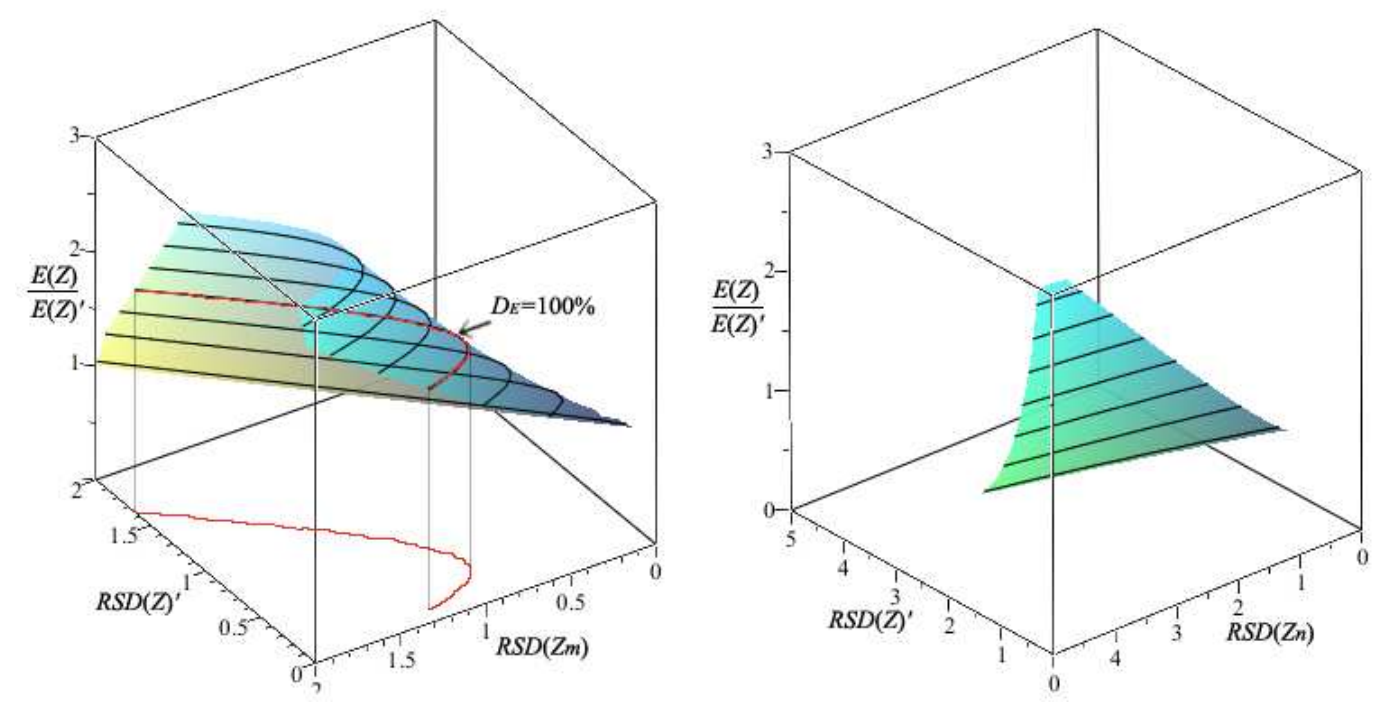

FIG.8

Hence, we found (1) For a certain $R S D(Z)^{\prime}, R S D\left(Z_{m}\right) \propto D_{E}$ derived from (III.2.3) and also from the simulation. In cases of $D_{E}>100 \%$, roughly all $R S D\left(Z_{m}\right)>100 \%$, thus the variety of $R S D$ has little impact on the feasibility of analysis but affects the efficiency of analysis - the larger the $R S D$, the smaller the $D_{P r}$. (2) When $D_{E}$ is low (<50\%), for a certain $D_{E}$, roughly $R S D\left(Z_{m}\right) \propto R S D(Z)$ ' since $R S D(Z)$ ' can not be too small in practice. In some cases of low $R S D\left(Z_{m}\right)$ their analysis may be not so feasible, which is acceptable. (3) Since in most cases $R S D\left(Z_{n}\right)>>100 \%$, the situation of $R S D(Z)>R S D\left(Z_{n}\right)$ can be ignored.

Finally, by the symmetry principle, the above argument also applies to case (1.b) and case (2). When those volume shifts to the left side, we will get the inverse result, thus vice versa. The reasonings of more complicated cases are similar because of the addition linearity of log-normal distribution. Thus, the overall analysis is feasible almost all the time on the condition of $R S D<100 \%$.

Theorem 2. (Volume Spectrum Theorem) In call auction, if the distribution of lot sizes is log-normal, then double volume spectrum of a current time window influences future stock prices in some definite way.

The span of volume shift in DVS ${ }^{\mathrm{C}}$ could be large or small. For instance, if some volume shifts from one side of $Z_{c}$ to another, the same volume on the other side will be canceled, which diminshes the $\left|\mathrm{TVD}^{\mathrm{C}}\right|$ and IAD. Since $x$ of such volume decrease greatly after the shift, it is quite possible that $P_{k}{ }^{\prime}<P_{k}$ or $P_{k}{ }^{\prime}>P_{k}$ as the above proof shows. However, if it shifts just to near partitions (or even inside its own), only the distribution of $\mathrm{DVS}^{\mathrm{C}}$ will change but TVD ${ }^{\mathrm{C}}$ 
and IAD will remain the same. Thereby, it is quite possible that $P_{k}{ }^{\prime}=P_{k}$ since $x$ changes only a little. $\operatorname{TVD}^{\mathrm{C}}\left(\delta_{c}\right)$ and IAD $(H)$ work as quality indexes in timbre evaluation since they have considerable impact on the expected return, so that they are denoted as central indexes. On the other hand, critical zero point $Z_{c}$ and critical scale $S_{c}$ are local indexes by analogy with style indexes in timbre evaluation. Another local index, symmetry degree of volume spectrum will be discussed in the next chapter.

\section{Proof in Continuous Trading}

Though continuous trading has different rules from those of call auction, two kinds of auctions must share the same characteristics otherwise they can not get along in one market. We observe that continuous trading can be divided into monotone subsections (periods of time in which the price monotonously increases or decreases) and each one of them is linearly equivalent to a call auction by turning its time sequence into price one of call auction. Therefore, all arguments of call auction also apply to continuous trading.

In art auction, the highest bidder must be the one who wants to get the work most. The situation is similar in call auction of stock market, a slight difference is that call auction is bidirectional. In any one of monotone subsections, the person placing the first market order is meant to be the one who wants to execute his/her order most, which implies he/she will be the highest bidder or lowest asker in an equivalent call auction.

Thumb Rule. Time priority principle is equivalent to price priority principle.

Consider a simple case: at one moment there are limited orders of ask placed in ascending order of price as $A_{1}, A_{2}, \ldots$ and a market order $B$ of bid whose lot size is much larger than the total lots at any one price level of above ask orders. Suppose the trader of $B$ is the one who wants to execute his/her order most, so that he/she will be the first to place an order for each market price. Thus the trader submits a series of buy orders as soon as possible in sequence of $B_{1}, B_{2}, \ldots$ that respectively match $A_{1}, A_{2}, \ldots$ until $B$ is completely filled. But according to the rule of continuous trading, he/she has an alternative operation: just submit a single order at an price high enough (no matter what the exact price is) so that it will be executed in the exactly same way as the previous one. The two operations are equivalent. The reverse case that a large market order of ask matches a series of limited orders of bid has the same result.

Now we take a general case for one subsection, say, monotone increasing. As call auction, consider a local range $[\alpha, \beta]$ of order prices, denote ask price as $S_{j}$ and its lot size as $T_{j}$, let market price is $p_{k}$ (= some $\left.S_{j}\right)$; sort filled ask orders in ascending order of prices: $s=\left[\left(S_{1}, T_{1}\right)\right.$; $\left.\left(S_{2}, T_{2}\right) ; \ldots ;\left(S_{m}, T_{m}\right)\right]\left(\alpha<S_{1}, \beta>S_{m}\right)$, which is a sequence of limit orders (orders with $S_{j} \leq p_{k}$ can be treated as limit orders with $p_{k}$ by the price priority principle, and orders with the same ask price are sorted by the time priority principle), and each filled order's volume weight

$$
w_{j}=T_{j} / \sum_{k=1}^{m} T_{k} .
$$


Denote bid price as $B_{i}{ }^{\prime}$ and its lot size as $C_{i}$. Because the subsection is monotone increasing, no limit bid orders can be filled. Those filled market orders (orders with $B_{i}{ }^{\prime} \geq p_{k}$ can be treated as market orders) can be rearranged in descending order of new prices $B_{i}$ according to submitting time $t_{i}, B_{i} \propto 1 / t_{i}$, which is based on the thumb rule. Thus we get a sequence of filled bid orders $b=\left[\left(B_{1}, C_{1}\right) ;\left(B_{2}, C_{2}\right) ; \ldots ;\left(B_{n}, C_{n}\right)\right]$ (no matter what the exact price of $B_{i}$ is but still high enough). The matching rule is the same as that of call auction, but the trading price is subject to the corresponding ask price. Suppose the last matched order $\left(B_{n}, C_{n}\right)$ is filled with $\left(C_{n}-R\right)$ lots. Hence, we get the average trading price of volume weighted

$$
\begin{gathered}
P^{-}=\sum_{j=1}^{m} w_{j} \cdot S_{j} \\
\text { and } \sum_{i=1}^{n} C_{i}-R=\sum_{j=1}^{m} T_{j}
\end{gathered}
$$

Comparing them with (III.1.1) and (III.1.2) gives us

Theorem 3. (Equivalent Auction Theorem) Continuous trading is linearly equivalent to call auction, which means double volume spectrum of a current time window has almost the same impact on future price $P$ of call auction and $P^{-}$of continuous trading.

\section{Applications of VSA}

\section{A. PDE of VSA}

Let us show the connection between (one-dimension) Brownian motion and diffusion euqation. Suppose on Y-axis a particle is located at $y=0$ when $t=0$, i.e. its state density function $\left.\rho(y, t)\right|_{t=0}=\delta(y), \delta(y)$ is Dirac delta function. Denote its diffusivity as $\sigma^{2} / 2$, and the diffusion equation is

$$
\frac{\partial \rho}{\partial t}=\frac{\sigma^{2}}{2} \frac{\partial^{2} \rho}{\partial y^{2}}
$$

thus its solution is

$$
\rho(y, t)=\frac{1}{\sqrt{2 \pi \sigma^{2} t}} \exp \left(-\frac{y^{2}}{2 \sigma^{2} t}\right)
$$

which means $\rho(y, t)$ is equivalent to the probability density of Brownian motion without drift, and $y$ can be defined as the expected return in VSA. Actually, (IV.1.1) is the passive heat conduction equation as well. But it implies that velocity of particle or heat conduction is infinite: they can move instantaneously to infinite distances as long as the probability exists. Of course it is an approximation to the reality - recall that empirical data of capital markets 
show the departures from normality. If we consider the limitation of information transmission speed - obviously, it will be the property not of state $y$ but of its transmission medium. For an instance of heat conduction, let $a^{2}=\sigma^{2} / 2$ : by Cattaneo-Vernotte Law, the heat transmission speed $c=a / \sqrt{ } \tau_{c}$, where $\tau_{c}$ is a relaxation factor (for isotropic mediums it is constant), and equation (IV.1.1) shall be modified as

$$
\begin{aligned}
& \frac{1}{a^{2}} \frac{\partial \rho}{\partial t}+\frac{1}{c^{2}} \frac{\partial^{2} \rho}{\partial t^{2}}=\frac{\partial^{2} \rho}{\partial y^{2}}, \\
& \text { or } \frac{\partial \rho}{\partial t}+\tau \frac{\partial^{2} \rho}{\partial t^{2}}=a^{2} \nabla^{2} \rho,
\end{aligned}
$$

where $c^{2}=a^{2} / \tau_{c}(y, t)$. For the PDE of VSA, the only possible transmission medium of $y$ is the volume or lot size $x$ of orders. Thus, we assume $\tau_{c}(y, t)$ is subject to DVS ${ }^{\mathrm{C}}$ by a mapping. The solution of (IV.1.2) implies a spectrum mixing wave and diffusion (normal distribution), which may explain asset bubbles and derive a new approach to option pricing. We will study this idea in a separate paper.

\section{B. Solution of VSA}

As we have shown in the previous chapter, VSA has two kinds of indicators: central and local indexes. The former is more important than the latter, but they are linked together. For example, a larger $S_{c}$ usually implies larger $\mathrm{TVD}^{\mathrm{C}}$ and IAD. Like that of timbre evaluation, symmetry degree of volume spectrum means the dominance of the highest column in DVS over other columns. It has two sub-indexes: $\mu_{l}$ left to $Z_{c}$ and $\mu_{r}$ right to $Z_{c}$, also including their places on $\mathrm{X}$-axis, respectively denoted as $w_{l}$ and $w_{r}$. Suppose the left and right highest columns of volume spectrum respectively has volume $V_{l}$ and $V_{r}$, we define $\mu_{l}=V_{l} / \delta_{c}$, and $\mu_{r}=$ $V_{r} / \delta_{c}$. With the same $S_{c}$, a large $\mu_{l}$ and $w_{l}$ may indicate an large investor is trading actively.

The application of VSA is an open question, which does not have such a single solution as BS formula or CAMP. Here we suggest a possible application of central index $\operatorname{TVD}^{\mathrm{C}}\left(\delta_{c}\right)$. As equations (III.1.1) and (III.3.1) show, if IAD is large enough, then the impact of $\mathrm{TVD}^{\mathrm{C}}$ on price is almost linear. Let us show a simple example of such application. Let $x$ be the day $\mathrm{TVD}^{\mathrm{C}}, y$ be the day return (calculated by VWAP), and take a historical time window $W_{0}$ as a benchmark, say 1-2 years. Thus we can get a regression line as $y=y_{0}+\lambda x$. Then we take another observation window $W_{i}$ that has $i$ days up to now $t_{0}$, in which there are two price curves: the actual price $p_{k}$ of day average and corresponding price $p_{k}$ ' of $\delta_{c}$ at $p_{k}\left(\delta_{k}\right)$. The latter is defined as $p_{k}{ }^{\prime}=\left(1+y_{k}\right) p_{k-1}$, where $y_{k}=y_{0}+\lambda \delta_{k}$. The difference $\varepsilon_{k}=p_{k}-p_{k}$ ' is called volume-price deviation (VPD). The investor could make decisions based on the changes of VPD, which depends on the length of $W_{i}$, for example, a long-term investor shall take a longer $W_{i}$ than that of a short-term investor. For the same price process, investors of different types may make different decisions. In Fig.9, suppose the short-term and long-term investor are considering to buy the stock. The former's observation window is $W_{i}$ and that of the latter is $W_{j}, i<j$. In $W_{i}, \varepsilon_{i}$ is stable and small enough, thus the short-term investor may buy - the price can rise in short term. While in $W_{j}, \varepsilon_{j}$ is getting larger and larger, hence for the long-term investor, buying this stock may take great risks. 
Moreover, $\lambda$ of VSA is much more stable than $\beta$ of CAPM. Though any slumps or surges will modify $\beta$ greatly, CAPM assume it is fixed and employ it in VAR (value at risk) - that is the major reason why CAPM is usually rejected by empirical data. In contrast, VSA defines risk as the deviation $\varepsilon$ rather than the volatility $\sigma^{2}$ in modern finance. Through the observation of deviation $\varepsilon$, VSA is able to predict slumps or surges. For example, if for most Dow Jones constituents we observe remarkably large deviation $\varepsilon$ in both short- and long-term windows, then it is quite possible that the financial crisis will occur soon after. In fact, hyper-issued currency from central bank's QE (quantitative easing) policy would enter the capital market in great part, but it does not affect all investors equally. The large investors who are well-connected to the banking system get the newly-created money first. Thus, in the early phase of bull market, $\delta_{c}$ is relatively high, but the price does not rise rapidly, i.e. the deviation $\varepsilon$ is usually negative. Then the money effect gradually extends to small investors, and $\delta_{c}$ begins to get lower while the price surges, i.e. the deviation $\varepsilon$ turns to be positive and gets larger and larger. The bubble continues to be blown up until it explodes. From 2008 to now, the major form of QE is buy-back since the benchmark rate is almost zero - the cost of debt is too cheap. We are sitting on the crater of corporate debts and waiting for the fatal hit. ETF probably triggers the problem of liquidity trap as CDO did in 2008. VSA may reduce losses, however, it can not stop the disaster.

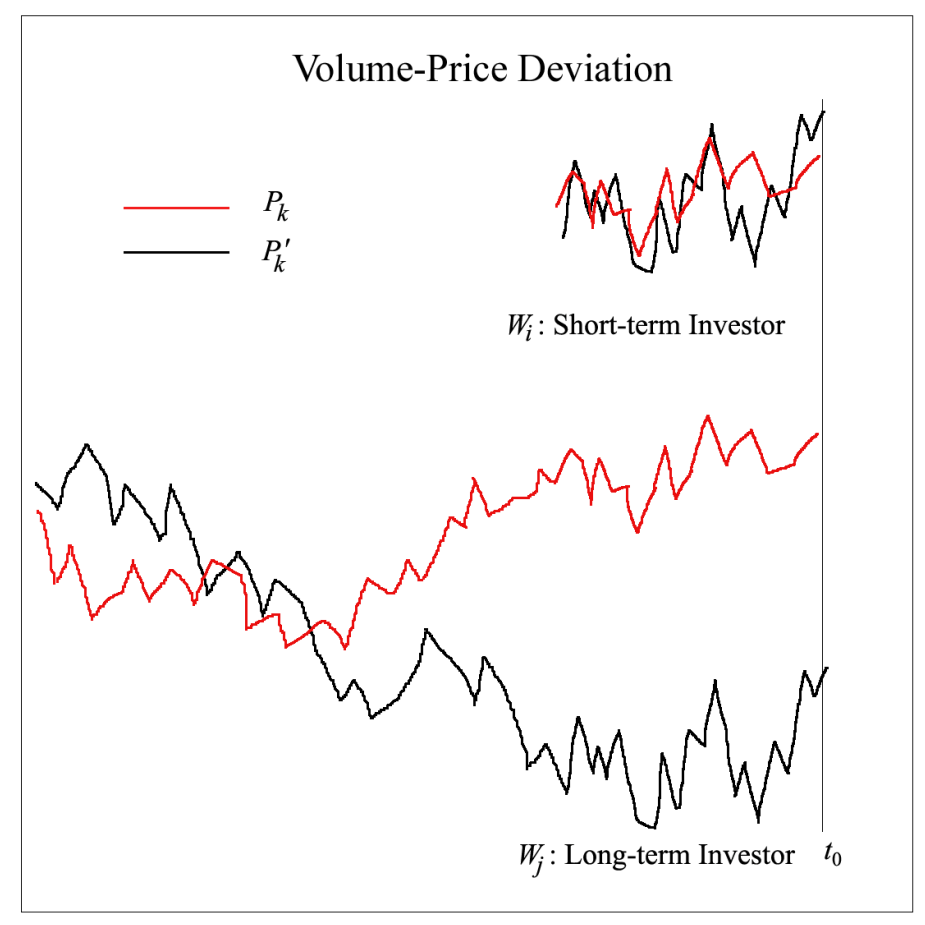

Fig.9

\section{Conclusion}

VSA has not only the ability of prediction, it also shows that information asymmetry leads to a hiberarchy of investment tenures. In fact, information asymmetry is the fundamental of trading in capital markets, otherwise, suppose all investors believe in EMH and take the 
strategy of buy-and-hold, e.g. ETF - the capital market will not exist at all because all investors are long-term ones and on one sells. For asset pricing, short-term trading is not speculation but an indispensable part of capital market. Some neo-classical economists think that the market with perfect information is the most efficient (has the largest size), and "governmental intervention [of reducing uncertainty] may increase the welfare of all parties." ${ }^{36}$ However, market solutions may do it better, such as VSA. Moreover, it is quite possible that the relationship between market efficiency and information symmetry is not monotone.

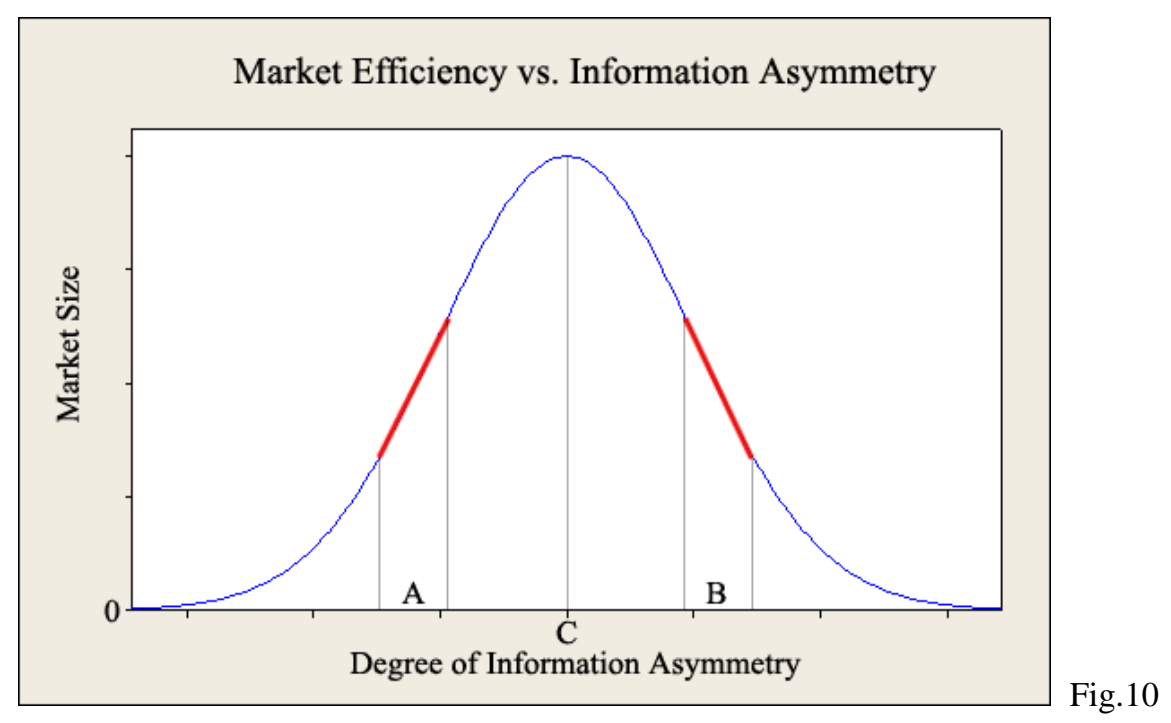

As Fig.10 shows, we classify information asymmetry as two types: type A and type B. The former represents the competition among producers. In this situation, the market size will enlarge when the degree of information asymmetry increases, e.g. patent protection, business innovation, scale economy etc., in other words, natural monopoly can promote the efficiency of market. However, when the degree of information asymmetry exceeds the critical point $\mathrm{C}$, for instance, erecting bars by governmental monopoly, the market size will shrink. On the contrary, type B denotes the trading in markets - it could be one-side trading between producers and consumers or double trading such as the capital market. In this case, the situation is opposite to type A. But whether in type A or B, perfect information or extremely asymmetric information does eliminate the market, just as "perfect competition" actually means there is no competition. For example, when many people were illiterate in ancient times, writing letters for others was a profitable job. But after the popularization of modern education, this occupation got to disappear today.

In Akerlof's case of "lemons", why used car dealers can make money? Because they have more information of second-hand cars than consumers. If every consumer is an expert of car mechanism, the market of dealers will disappear (but the market of used cars still exists) - it is almost impossible in reality since in general the market is always within the red section of Fig.10. Similarly, EMH is an illusion as well. Buy-back is a good example: the company itself must possess more information than outside investors - such asymmetry can not be

\footnotetext{
${ }^{36}$ George A. Akerlof, The Market for "Lemons": Quality Uncertainty and the Market Mechanism, The Quarterly Journal of Economics, Vol.84, No.3. (Aug., 1970), P.488.
} 
eliminated at all. However, bubbles do not come from buy-back - companies have the right to buy back - financial or economic crisis is created by the government, the central bank and their related players (e.g. executives of companies), because they can capitalize on distorting the natural hiberarchy of capital market.

Another observation is that the assumption of utility maximization does not work in the field of capital market. Utility function does not apply to security since it is not consumable. Moreover, even in the researches of one-side auctions, psychological utility is excluded so that every players has the same utility function ${ }^{37}$ - obviously it does not make sense. As we have shown in chapter I, the concept of static equilibrium does not apply to the dynamic asset pricing.

In sum, both EMH and game theory address economic actions as a paradigm like those in physics or mathematics. Such paradigm, though modified in applications, however, is far away from the reality since people are not uniform molecules. In stead, VSA may open a new door to reveal the essence of human actions in capital markets.

${ }^{37}$ Myerson, Optimal Auction Design, P.61. 